\title{
Identity and Indeterminacy in -ever Free Relatives
}

\author{
Daphna Heller and Lynsey Wolter \\ University of Rochester
}

\section{Introduction}

A free relative (FR) containing -ever is felicitous only when the speaker doesn't know, or doesn't care about, the identity of the entity denoted. For example, (1a) is felicitous if the speaker doesn't know if Mary is cooking pea soup or spinach (although he may know that the thing Mary is cooking is green). Conversely, if the speaker knows that Mary is cooking pea soup, it would be infelicitous to use the -ever FR. In a felicitous use of (1b), where whatever combines with the head noun soup, the speaker does not know what kind of soup Mary is cooking. Example (2), meanwhile, is infelicitous if the speaker can name the person who arrived last.

(1) a. Whatever Mary is cooking uses onions. (Dayal 1997: ex. 27)

b. Whatever soup Mary is cooking uses onions.

(2) Whoever arrived at the party last brought the soup.

Standard FRs, i.e. DP-like maximal FRs that are headed by a plain whword (Caponigro 2003), are like -ever FRs in that they also suggest indeterminacy with respect to the entity satisfying the content of the FR. However, standard FRs differ from -ever FRs in that they do not require this indeterminacy. Thus, if (3) is uttered out of the blue, the addressee is likely to infer that the speaker does not know whether Mary is cooking pea soup or spinach. However, the speaker may cancel this inference by explicitly naming the thing Mary is cooking, as in (4a). By contrast, the speaker of (1a) cannot felicitously name what Mary is cooking, as shown in (4b).

(3) What Mary is cooking uses onions.

(4) a. What Mary is cooking, namely pea soup, uses onions.

b. *Whatever Mary is cooking, namely pea soup, uses onions.

In this paper we ask what conditions allow a felicitous use of an -ever FR. Since-ever FRs are felicitous when the speaker doesn't know, or doesn't care about, the identity of the entity satisfying the content of the FR, i.e. when the entity is not identified, we ask what conditions count as non-identification of an entity. In the next section we discuss Dayal's (1997) suggestion that nonrigidity is nonidentification, and hence -ever FRs can be used when the denotation of the FR is

We would like to thank Greg Carlson, Christine Gunlogson, and David Braun for their insightful comments at different stages of this work. We also thank the audience at SALT 18, and especially Jason Stanley, Philippe Schlenker, and Bernhard Nickel, for much helpful feedback. 
nonrigid. We present cases of rigidity where an -ever FR is nonetheless licensed as well as cases of nonrigidity where an -ever FR is infelicitous; these pose a problem for an account which takes rigidity to be the same as identification. In section 3 we furthermore show that the rigidity-based account cannot be amended by adding a second condition; this leads us to conclude that the source of the problem is our assumptions about identity and identification more generally. In section 4 we present an alternative approach to identity and identification, adopting Gupta's (1980) logic of common nouns, which allows us, in section 5, to present our analysis of the indeterminacy requirement on -ever FRs. We conclude that the felicity condition on -ever FRs illuminates the nature of identity and identification in natural language.

\section{The Relation Between Rigidity and Identification}

Our point of departure is Dayal's (1997) proposal that the indeterminacy of -ever FRs, i.e. their requirement that their denotation not be identified, amounts to the nonrigidity of the FR denotation across a set of possible worlds. Dayal follows Jacobson (1995) in treating FRs as definite descriptions (an analysis we also adopt), and proposes that -ever FRs require nonrigidity with respect to the speaker's epistemic state, as paraphrased in (5).

Condition $\mathbf{R}$ Given an -ever FR denoting $\lambda w . \imath x\left[P_{\langle s,\langle e, t\rangle\rangle}(w)(x)\right]$, there exist worlds $w^{\prime}, w^{\prime \prime}$ epistemically accessible to the speaker such that $\imath x\left[P\left(w^{\prime}\right)(x)\right] \neq \imath x\left[P\left(w^{\prime \prime}\right)(x)\right]$.

Subsequent work by von Fintel (2000) and Tredinnick (2005) has challenged the assumption that the locus of variation for an -ever FR is always the set of worlds epistemically accessible to the speaker. For example, they note that "indifference" readings, such as the most salient reading of (6), are better characterized as involving non-identification of the FR denotation relative to a counterfactual modal base. However, von Fintel (2000) and Tredinnick (2005) maintain the assumption that identification is rigidity and that -ever FRs therefore require nonrigidity relative to some set of worlds.

(6) I grabbed whatever tool was handy. (von Fintel 2000: ex. 17)

In this paper, we are concerned with the nature of (non)identification rather than with the locus of variation. We continue to work with the "ignorance" readings that Dayal focuses on, where the locus of variation is related to the speaker's beliefs. This is because these ignorance readings lend themselves to the construction of contexts in which we can test what kinds of information count as identifying an entity. ${ }^{1}$ While our discussion does not include all the readings found in -ever

\footnotetext{
${ }^{1}$ Condoravdi (2008) and von Fintel (2000) observe that even "ignorance" readings are not necessarily anchored to the speaker's beliefs, but rather to the beliefs of one of the interlocutors. For example, an -ever FR like (i) can be used in a "teasing" context where the speaker, but not the addressee, can identify what is being cooked.
} 
FRs, our findings about the nature of indeterminacy must be incorporated into any complete theory of -ever FRs.

The rest of this section considers the felicity of -ever FRs in different situations. While there are cases in which nonrigidity indeed coincides with nonidentification (section 2.1, Case A), we also present cases where rigidity coincides non-identification (section 2.2, Case B) as well as cases where nonrigidity coincides with identification (section 2.3, Case C).

\subsection{Case A. Proper Names: Rigidity is Identification}

Dayal's Condition R accounts for the fact that (7) (repeated from 2) is infelicitous if the speaker knows that Alex arrived last (remember we are focusing on the ignorance reading).

(7) Whoever arrived last at the party brought the soup.

Suppose that Austin has overheard that Alex arrived last at the party. Austin has also tasted everything but the soup, which appeared late, so he infers that Alex brought the soup. However, he has never met Alex and doesn't even know whether Alex is a man or a woman. This is a situation where Austin only knows that the person who arrived last is named Alex; he knows no other properties of the person who arrived last. In this situation, Austin cannot felicitously utter (7). This demonstrates that simply establishing a name for the entity satisfying the content of an -ever FR counts as identifying the entity, and precludes the use of the FR.

The received wisdom about proper names is that they are rigid designators (Kripke 1982). So the logic of Condition $\mathrm{R}$ is this: knowing a name allows the entity satisfying the content of the FR to be rigidly designated, resulting in a lack of variation (or the absence of the required indeterminacy) across the speaker's epistemically accessible worlds. This violates Condition $\mathrm{R}$ and the -ever FR is correctly ruled out. ${ }^{2}$

\subsection{Case B. Demonstrative Pronouns: Rigidity Without Identification}

Suppose Becca enters the kitchen and sees Bob stirring a pot. Becca cannot say what dish Bob is cooking, but she can point out the stuff that he is stirring - that is, she's in a position to use a demonstrative pronoun, as in (8).

(8) Bob is cooking that.

(i) There's a lot of garlic in whatever it is I'm cooking! (von Fintel 2000: ex. 7)

We suspect that the locus of variation for "ignorance" readings may ultimately depend on the mutual public commitments of the interlocutors, in the sense of Gunlogson (2001). Though we will continue to describe -ever FRs as requiring indeterminacy with respect to the speaker's epistemic state, this should be taken as a simplification for expository purposes.

${ }^{2}$ Note that names are standardly assumed to be metaphysically rigid, not epistemically rigid. Condition $\mathrm{R}$ thus requires an additional assumption connecting the (metaphysical) rigidity of names with the (apparently epistemic) locus of variation. 
If Becca can infer from the smell that one ingredient is onions, she can felicitously utter (9).

(9) Whatever Bob is cooking uses onions.

This example demonstrates that being in a position to use a demonstrative pronoun does not rule out the felicitous use of an -ever FR. Demonstrative pronouns, like proper names, are standardly assumed to be rigid designators (Kaplan 1989), so given our previous observations about names, this is quite surprising. Condition $\mathrm{R}$ predicts that a speaker who is in a position to use a demonstrative pronoun - just like a speaker who is in a position to use a proper name — should not be able to utter an -ever FR felicitously, but in this case this is the wrong prediction.

Note that the same facts are found with -ever FRs containing nominal heads. If Becca can point at the stuff that Bob is cooking and knows that it is soup, but doesn't know what kind of soup it is, she can felicitously utter (10).

(10) Whatever soup Bob is cooking uses onions.

In sum, in the examples we have just seen, the denotation of the -ever FR is rigid across the relevant set of possible worlds, and yet - contra the prediction of Condition $\mathrm{R}$ - the -ever FR is felicitous. In other words, this is a case of rigidity that does not seem to result in identification. If we want to maintain Condition $\mathrm{R}$ and still account for the facts in Case B, we would have to argue that being able to use a name induces rigidity in the relevant set of worlds, and being able to use a demonstrative pronoun does not. This presents a serious challenge to Condition $\mathrm{R}$ in accounting for the felicity of -ever FRs.

\subsection{Case C. Nominal Predicates: Identification without Rigidity}

Suppose Christina knows that Carlos is cooking soup, though she doesn't know anything else about the stuff Carlos is cooking and cannot point out the relevant soup (perhaps she heard Carlos yell from the kitchen that he is cooking soup). In this context, Christina cannot felicitously utter (11) on an ignorance reading.

(11) Whatever Carlos is cooking will be delicious.

Condition $\mathrm{R}$ does not predict this fact. Note that Christina is not in a position to refer rigidly to the entity satisfying the content of the FR, either by using a demonstrative pronoun or by using a proper name. Since the denotation of the FR is nonrigid across the relevant set of possible worlds, Condition R predicts (11) to be felicitous. All Christina knows about the entity satisfying the FR, beyond the content of the FR itself, is that it has the property of being soup. Yet knowing this property apparently counts as identification, as it precludes the use of an -ever FR. ${ }^{3}$

Not all properties identify. Suppose that Christina doesn't know what Carlos is cooking because he hasn't told her, but she can perceive a strong odor coming out

\footnotetext{
${ }^{3}$ The fact that knowing a nominal property counts as identification and precludes the use of an -ever FR was originally noticed in Heller (2005) and independently in Condoravdi (2008).
} 
of the kitchen and knows Carlos is an excellent cook. In this context, Christina can felicitously utter (11), which will be a truthful statement if she is a fan of pungent foods. That is, knowing that the entity satisfying the FR has the property of being smelly does not preclude the felicitous use of the FR. The adjectival property smelly does not seem to identify the entity, contrasting with the nominal predicate soup, which does. More generally, nominal predicates, but not adjectival or verbal ones, count as identifying. The speaker of (11) can know that the dish Carlos is cooking is bland, but not that it is stir-fry. She can know that it is burning, but not that it is casserole. And so on.

The careful reader has probably noticed that we have already presented cases where a nominal property is known and yet an -ever FR is felicitous. These are cases where the nominal property is explicitly given in the FR. The speaker of (12) below knows that Carlos is cooking soup. In this case, the indeterminacy requirement applies to a more specific nominal property, e.g. corn chowder, to the entity satisfying the content of the FR.

Whatever soup Carlos is cooking will be delicious.

The relevant kind of indeterminacy, then, has to do with nominal categories that are not entailed by the content of the FR - hardly a surprising constraint.

More interesting is the fact that there is a preference for variation in basiclevel nominals, in the sense of Brown (1965). Notice that we are likely to assume that the speaker of (11) knows that Carlos is cooking food; ${ }^{4}$ the indeterminacy is with respect to nominals that are more specific than food, e.g. soup or casserole, but not with respect to extremely specific nominals, e.g. corn chowder or pea soup. These contrasts relate to very general preferences for certain nominals in referring expressions. Brown (1965) observes that although there are different lexical nouns which apply to the same object, some nouns are most commonly used when talking about these objects. For example, an entity that belongs to the class of food, soup and corn chowder will most frequently be referred to as a soup. Brown suggests that this is because the information expressed in basic-level nominals, such as soup, is in most cases the most important or relevant for the purposes of communication - more so than the information that this entity belongs to the class of food, which is the superordinate-level nominal, or to the class of corn chowder, which is the subordinate-level nominal. While the reason for preferring basic-level nominals remains an open question (see Cruse 1977 for an analysis of these facts using Gricean maxims), their cognitive priority is intuitively clear. It is therefore not surprising to find that establishing a basic-level nominal, as opposed to a superordinate- or subordinate-level nominal, is what counts as identification, and that in the unmarked case, such as (11), -ever FRs require indeterminacy with respect to basic-level nominals.

Consider (13) below, which further exemplifies the preference for indeterminacy at the basic level. This sentence is felicitous in a context where the speaker

\footnotetext{
${ }^{4}$ While this property might be strongly implied by the content of the FR in (11), it is not in fact entailed by it, as Carlos could potentially be cooking soap or playdoh.
} 
doesn't know anything about Meghan's entry - it could have been a sheep or a pie or a watercolor painting. Interestingly, (13) is also felicitous in a context where it has been previously established that Meghan entered some kind of food. In this case food is not at all implied by the content of the FR, again showing that if the speaker knows that a certain superordinate-level nominal applies to the entity denoted, this does not count as identification. If the speaker can apply a basic-level nominal like pie (or sheep or painting), the sentence is no longer felicitous.

(13) Whatever Meghan entered in the county fair won a blue ribbon.

What we observe, then, is that indeterminacy at the basic level is preferred, and it is only when the content of the FR entails a basic-level nominal, as in (10) or (12), that we observe indeterminacy with respect to subordinate-level nominals. Note that even in those cases the variation must be as close to the basic level as possible: (12) requires the speaker not to know whether Carlos is cooking pea soup or lentil soup. If the speaker knows that Carlos is cooking pea soup but entertains the possibility that he is cooking various subtypes of pea soup, (12) is infelicitous.

\subsection{Taking Stock}

Let us briefly summarize what we have seen so far. Knowing a proper name or a nominal predicate both count as identification and thus preclude the use of an -ever FR. The relevant nominals are subject to two constraints: (i) they are not entailed by the content of the FR, and (ii) they are as close to the basic level as possible. By contrast, knowing an adjectival or a verbal property, and, more surprisingly, being able to refer to an entity with a demonstrative pronoun, do not count as identification, and allow the use of an -ever FR.

These facts present a problem for Condition $\mathrm{R}$, which does not distinguish among properties and incorrectly predicts that knowing a nominal property never counts as identifying an entity. The challenge, then, is to find a new way of characterizing identification in which being able to apply a name or a nominal property would count as identification, and being able to use a demonstrative pronoun or apply a verbal or adjectival property would not.

\section{Against "Rigidity Plus"}

At this point the obvious direction is to try to amend Condition $\mathrm{R}$ by adding a second condition that requires variation in certain nominal properties. In this section we will consider such an approach and show why it does not work. Investigating this dead end is instructive, because it reveals that the source of our problem is quite fundamental: the notion of identity and identification implicit in our discussion so far simply does not provide the tools for a satisfying account of the indeterminacy of -ever FRs. 
Condition $\mathrm{N}$ in (14) below requires variation with respect to the predicates that apply to the denotation of the FR in each relevant world.

Condition N. Given an -ever FR denoting $\lambda w . l x\left[P_{\langle s,\langle e, t\rangle\rangle}(w)(x)\right]$, there exists no $Q_{\langle s,\langle e, t\rangle\rangle}$

such that for every $w$ in the epistemic alternatives of the speaker $Q(w)(\operatorname{lx}[P(w)(x)])=1$.

Like all quantified statements, the domain of properties over which $\mathrm{Q}$ ranges is restricted by the context - this is what limits the properties under consideration to those that are as close to the basic level as possible but not entailed by the content of the FR. In addition, however, we must add a restriction that $\mathrm{Q}$ ranges over nominal properties and not verbal or adjectival ones. Unlike the pragmatically motivated restriction to basic-level nominals, this restriction is nothing but a stipulation.

If we are willing to accept this stipulation, though, Condition $\mathrm{N}$ does some useful work. In particular, in a context where the speaker knows a nominal property of the entity denoted by an -ever FR (Case C), this condition correctly rules out the use of an -ever FR. At first glance, Condition $\mathrm{N}$ is a promising characterization of one aspect of the indeterminacy introduced by -ever FRs.

Unfortunately, when we look more closely, it turns out that no combination of Condition $\mathrm{R}$ and Condition $\mathrm{N}$ can accounts for the full set of facts. We have already seen that Condition $\mathrm{R}$ alone cannot account for all of the facts. Neither can Condition $\mathrm{N}$ alone: although this condition correctly predicts an -ever FR to be felicitous if the speaker can point out the denotation of the FR (Case B), and correctly rules out an -ever FR when the speaker can ascribe a nominal property to the denotation of the FR (Case C), Condition $\mathrm{N}$ fails to rule out an -ever FR when the speaker can apply a proper name to the denotation of the FR (Case A).

An obvious alternative is that -ever FRs are subject to both Condition $\mathrm{R}$ and Condition N. This approach correctly rules out all of the infelicitous uses of -ever FRs that we have observed. If the speaker can name the entity satisfying the content of the FR (Case A), the FR is ruled out by Condition R, and if the speaker can ascribe a nominal property to the entity (Case C), the FR is ruled out by Condition N. However, since the -ever FRs are still subject to Condition R, it incorrectly rules out cases where a speaker is in a position to point out the entity satisfying the FR and to refer to it with a demonstrative pronoun (Case B).

The last alternative we consider is that -ever FRs are licensed by satisfying either Condition $\mathrm{R}$ or Condition $\mathrm{N}$. This would correctly allow the felicitous uses of -ever FRs that we have observed. In particular, it would correctly predict that a speaker who can point out the denotation of an -ever FR (Case B) can still use the $\mathrm{FR}$, as long as she cannot also ascribe a nominal property to it. But this analysis is far too liberal, allowing many infelicitous uses. It predicts an -ever FR to be felicitous when the speaker can name the denotation of the FR (Case A), as long as the speaker does not also ascribe a nominal property to it, and it predicts an -ever FR to be felicitous when a speaker can ascribe a nominal property to the denotation of the FR (Case C), as long as the speaker does not name it. Table 1 summarizes how Condition $\mathrm{R}$ and Condition $\mathrm{N}$ deal with the different cases we present. 


\begin{tabular}{|l|c|c|c|}
\hline & $\begin{array}{c}\text { Case A } \\
\text { proper names }\end{array}$ & $\begin{array}{c}\text { Case B } \\
\text { demonstrative pronouns }\end{array}$ & $\begin{array}{c}\text { Case C } \\
\text { nominals }\end{array}$ \\
\hline Condition R & $\checkmark$ & $*$ & $*$ \\
\hline Condition N & $*$ & $\checkmark$ & $\checkmark$ \\
\hline Condition $\mathrm{R} \wedge$ Condition N & $\checkmark$ & $*$ & $\checkmark$ \\
\hline Condition R $\vee$ Condition N & $*$ & $\checkmark$ & $*$ \\
\hline
\end{tabular}

Table 1: How Condition R and Condition $\mathrm{N}$ deal with the different cases

In sum, the rigidity-as-identification approach cannot be fixed by simply adding a second condition that deals with variation in nominal properties. To account for the indeterminacy in -ever FRs, we need to rethink our conception of identity and identification. We turn to this task next.

\section{Rethinking Identity and Identification: Gupta's Logic of Common Nouns}

It is well known that nominal predicates differ from verbal and adjectival predicates both in their syntactic distribution and in the information they encode. As illustrated in (15-16), only nominal predicates occur as the complement of quantifiers.

a. Every soup was equally tasty.

b. There were at least five statues in the garden.

a. *Every smelly turned out to be tasty.

b. *There were at least five boiling on the stove.

Standardly, all predicates, regardless of their syntactic category, are assumed to denote functions from worlds into sets of entities. Under this analysis, semantic type cannot be the explanation for the contrast in (15-16). This motivated Gupta (1980) to consider the possibility that the denotation of nominal predicates is at a different type than verbal and adjectival predicates.

The semantic type proposed by Gupta (1980) has to do with the information expressed in nominals. In particular, nominals are special in that they provide criteria for judging whether two things, encountered at different times or in different hypothetical situations, are the same thing or different things. That is, nominals provide the criteria relevant to (transworld) identity and identification. The relevant intuitions are particularly salient for nominals that occur with same and different, as in (17).

(17) Michelangelo actually carved David from a certain block of marble.

a. David could have been made of a different piece of marble.

b. (Pointing at David) This piece of marble could have been a different statue. 
Although (17a) and (17b) are easy to understand, these sentences are surprisingly difficult to analyze in the type of model typically used by linguists. Consider Figure 1, where $w *$ is the actual world.

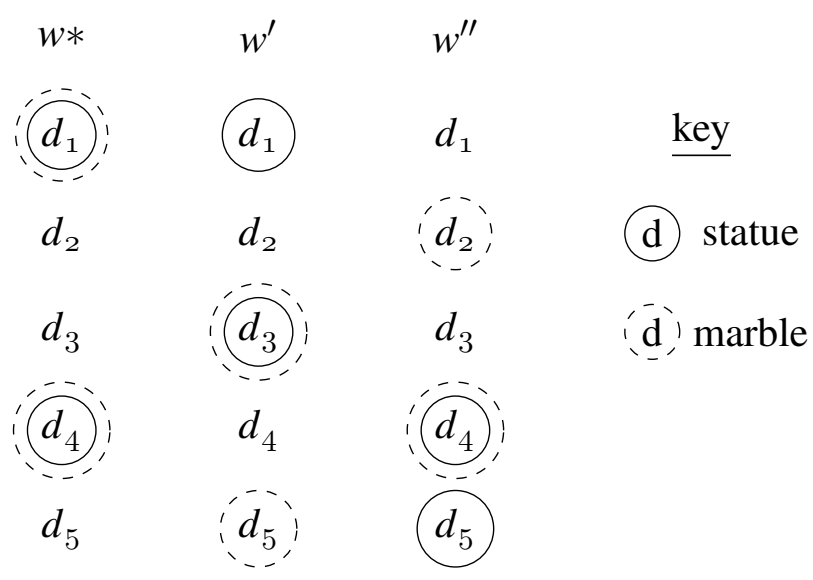

Figure 1: Information represented in a typical model

Suppose that $d_{4}$ in $w *$ is David. In order for (17a) to be true, there has to be a world in which David is made of a different piece of marble. We can find David in other worlds $-d_{4}$ is the same entity throughout — and verify that $d_{4}$ is made of marble in $w^{\prime \prime}$, but we don't have a way to represent whether $d_{4}$ is made of the same marble in $w *$ and $w^{\prime \prime}$ or of different pieces of marble in the two worlds. Similarly, if $d_{4}$ in $w *$ is the referent of the demonstrative in (17b), then to show that (17b) is true, we need to find another world where $d_{4}$ is made into a different statue. We can easily verify that $d_{4}$ is a statue in $w^{\prime \prime}$, but we don't have a way to represent whether $d_{4}$ is the same statue in $w *$ and $w^{\prime \prime}$ or different statues in the two worlds. The problem rests in the fact that a typical model has only a single, trivial principle of identity where every entity is identical to itself, so being the same statue cannot be distinguished from being the same marble.

The solution proposed by Geach $(1962,1972)$, and elaborated by Gupta (1980), is to replace the single, trivial principle of identity with multiple, nontrivial principles of identity, as illustrated in Figure 2 (again, $w *$ is the actual world).

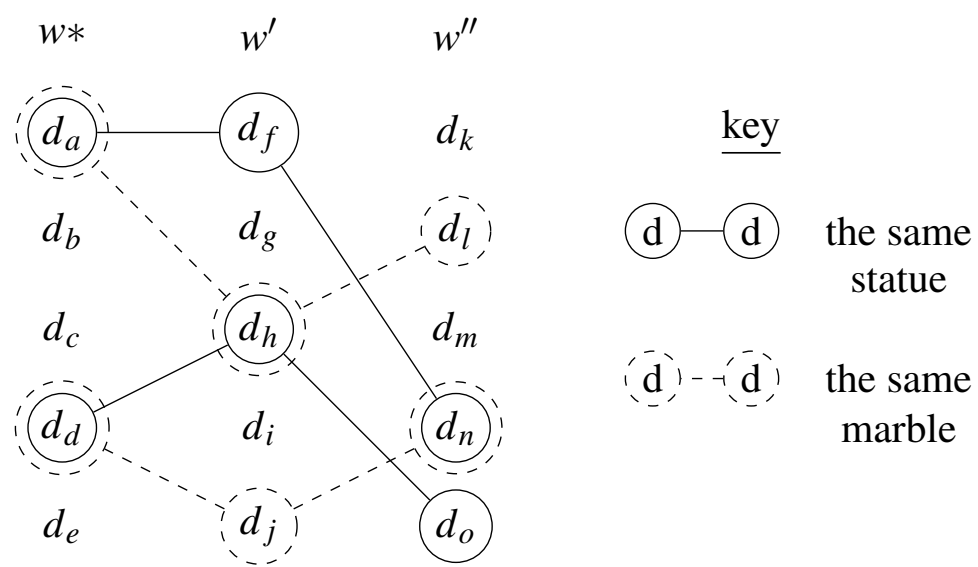

Figure 2: Information represented in a Geach-Gupta model 
The principles of identity for statues and pieces of marble are now different: the solid lines connect the corresponding statues across worlds, and the dashed lines connect the corresponding pieces of marble across worlds. This allows us to represent a state of affairs in which $d_{a}$ in $w *$ is the same statue as $d_{f}$ in $w^{\prime}$, but not the same piece of marble, while $d_{a}$ in $w *$ is the same piece of marble as $d_{h}$ in $w^{\prime}$, but not the same statue. Note that while the subscripts in Figure 1 are meaningful - they represent the (trivial) principle of identity - the subscripts in Figure 2 are not part of the model and are used here only for ease of reference.

In a Geach-Gupta model, then, nouns differ from other predicates in that, in addition to providing a principle of application, i.e. saying whether an entity has a certain property in a world, they also provide a principle of identity, i.e. information that tracks the entity across possible worlds and allows identifying it in different worlds. Gupta (1980) proposes that a "common noun" (roughly what we would consider to be the syntactic complement of a determiner) denotes a sort: a function from worlds into sets of individual concepts ${ }^{5}$ at type $\langle s,\langle\langle s, e\rangle, t\rangle\rangle$. To illustrate, consider the denotation of statue as a sort.

$\llbracket$ statue $\rrbracket^{M, w *, g}$ (type $\langle s,\langle\langle s, e\rangle, t\rangle\rangle$ ) The function from worlds $w$ to sets of individual concepts $I$ such that:

a. For each individual concept $i$ in $I, i(w)$ is a statue;

b. for all $w^{\prime}, w^{\prime \prime}:$ if $i\left(w^{\prime}\right)$ and $i\left(w^{\prime \prime}\right)$ are defined, then $i\left(w^{\prime}\right)$ is the same statue as $i\left(w^{\prime \prime}\right)$;

c. The sort is separated: for all individual concepts $i, i^{\prime}$ that belong to $\llbracket$ statue $\rrbracket^{M, w *, g}$ at world $w$, if at some $w^{\prime}, i\left(w^{\prime}\right)=i^{\prime}\left(w^{\prime}\right)$ then $i=i^{\prime}$.

Suppose we interpret the sort statue relative to $w$; this will give us the set of statue individual concepts in $w$. The values of these individual concepts relative to $w$ are the statues in $w$ - this is the principle of application shown in (18a). In addition, these individual concepts track the corresponding statues across other possible worlds. This is the principle of identity shown in (18b). Finally, by definition, the set of individual concepts is separated: two distinct statues in one world correspond to two distinct statues in other worlds and will never collapse into a single statue. This is shown in $(18 \mathrm{c}) .^{6,7}$

Before we turn to our analysis of the indeterminacy of -ever FRs, it should be noted that not all nominals are analyzed by Gupta as having a sort denotation, as some nominals do not seem to provide principles of identity. One nominal which does not denote a sort is thing. This can be illustrated if we compare a situation where you need to follow the instruction in (19a) with a situation where you need

\footnotetext{
${ }^{5}$ Throughout the paper, the term individual concept is used in a technical sense to mean a function from worlds into entities at type $\langle s, e\rangle$. It should not be taken to convey a concept, or to return individuals as opposed to other entities.

${ }^{6}$ Gupta (1980) adopts a stronger requirement where separation is not relativized to a world $w$ : see p. $29 \mathrm{ff}$ for discussion.

${ }^{7}$ Separation becomes more complicated when we consider mass nouns, where we need to track certain quantities across possible worlds. We abstract away from this complication here.
} 
to follow the instruction in (19b). While for the first case it is straightforward to decide which entities to count (and thus different counters will arrive at the same number), counting things is more open-ended: one has to decide which entities to count (and thus different counters may arrive at different results).

a. Count the people in this room.

b. Count the things in this room.

Gupta argues that if we take thing to denote at type $\langle s,\langle\langle s, e\rangle, t\rangle\rangle$ (the type of sorts), the resulting denotation will not be separated. That is, while we might on some occasions be able to identify two entities as "the same thing," there will also be occasions where one thing in one world corresponds to two things in another world. For example, consider two people who follow the instruction in (19b). The first person decides to count the desktop computer as one thing. The second person chooses to count the monitor, the keyboard and the mouse as separate things. Intuitively, both people have made reasonable decisions, but the result is that one thing in the first counting situation corresponds to three things in the second counting situation. This is a case where thing is not separated, and an illustration of why Gupta concludes that it does not in fact denote a sort.

\section{Sorts and Non-identification in -ever FRs}

The principles of identity discussed by Geach and Gupta provide the tools we need to present a new characterization of non-identification that will define the indeterminacy requirement on -ever FRs. Our proposal is simple: establishing which principle of identity, i.e. which sort, tracks an entity across worlds is what counts as the identification of that entity. Since for an -ever FR to be felicitous the entity denoted by the content of the FR must not be identified, there should be no sort that applies to that entity. More formally, we propose that -ever FRs are subject to Condition $\mathrm{G}$ below, which requires that no sort would hold of the intension of the FR in the relevant set of possible worlds (e.g. the epistemic state of the speaker under Dayal's analysis of the ignorance reading). If a principle of identity has been established for an entity, such a G would exist, and an -ever FR would be ruled out.

(20) Condition G. Given an -ever FR denoting $\lambda w . \imath x\left[P_{\langle s,\langle e, t\rangle\rangle}(w)(x)\right]$, there exists no $G_{\langle s,\langle\langle s, e\rangle, t\rangle\rangle}$

such that for every $w$ in the epistemic alternatives of the speaker $G(w)(\lambda w . l x[P(w)(x)])=1$.

Like Condition N (see again 14), Condition G rules out -ever FRs when the speaker can apply a nominal predicate to the entity denoted by the FR. The immediate advantage of Condition $\mathrm{G}$ is that we no longer need to stipulate that verbal and adjectival predicates are disregarded. This falls out of Gupta's semantics: verbal and adjectival predicates do not denote sorts, because they do not provide a principle of identity. This sets the stage for a new conception of identification: we 
link non-identification in -ever FRs to the notion of transworld identity developed in the philosophical literature for independent reasons.

To complete the account we must consider the relation of proper names and demonstrative pronouns to Gupta's sorts. We turn to proper names next.

\subsection{Names as Sorts}

Although Gupta (1980)'s analysis concentrates on the interpretation of common nouns, we will see in this section that proper names share those properties of nouns that led Gupta to suggest a sort denotation, and thus, we argue, proper names also denote sorts. First, note that names, like nominals, can occur as the complements of quantifiers.

(21) a. Every Susan that Katie met at the party shook her hand.

b. There are at least five Davids in my family.

More importantly, names are like nouns in that they express principles of identity. Again, we combine the items we are interested in with same and different to test our intuitions about the principle of identity. In (22), we see that names express the kind of information that is used to judge whether two things encountered at different times or in different situations are the same or not.

(22) a. This is the same Susan we saw at the ceremony.

b. This is a different David than the one we talked about earlier.

A sort denotation of a name, as in (23) below, allows us to capture the idea that names are like common nouns in expressing a principle of identity.

(23) $\llbracket$ Susan $\rrbracket^{M, w *, g}$ : (type $\left.\langle s,\langle\langle s, e\rangle, t\rangle\rangle\right)$ the function from worlds to sets of individual concepts $I$ such that:

a. For all individual concepts $i$ in $I, i(w)$ is Susan

b. for all $w^{\prime}, w^{\prime \prime}:$ if $i\left(w^{\prime}\right)$ and $i\left(w^{\prime \prime}\right)$ are defined, then $i\left(w^{\prime}\right)$ is the same Susan as $i\left(w^{\prime \prime}\right)$

c. the sort is separated: for all individual concepts $i, i^{\prime}$ that belong to $\llbracket$ Susan $\rrbracket^{M, w *, g}$ at world $w$, if at some $w^{\prime}, i\left(w^{\prime}\right)=i^{\prime}\left(w^{\prime}\right)$ then $i=i^{\prime}$.

Clause (23a) captures the principle of application expressed by the name. That is, (23a) reflects the fact that someone who comprehends the name Susan knows that it is in principle possible to separate entities in the actual world into those that are Susan and those that are not. The difference here between names and common nouns is that the principle of application associated with a proper name is arbitrary or causal (cf. Kripke 1982), while the principle of application associated with a common noun may be grounded in one or more non-arbitrary properties.

Clause (23b) gives the principle of identity associated with the proper name Susan. This reflects the fact that someone who comprehends Susan knows that 
it is in principle possible to link corresponding Susans across different situations, worlds, or times. Finally, (23c) is the separation condition that ensures that two distinct Susans in one world will not collapse into a single Susan in another world. Interestingly, this analysis differs from the account of proper names given within a standard model in that it does not require there to be a unique Susan. In a standard model, having more than one Susan would compromise the rigidity of the proper name. But the analysis of names as sorts does not have this problem because in a Geach-Gupta model, each individual named Susan in the actual world is tracked across possible worlds by a distinct individual concept, and the denotation of the proper name simply collects these individual concepts, as shown in Figure 3.

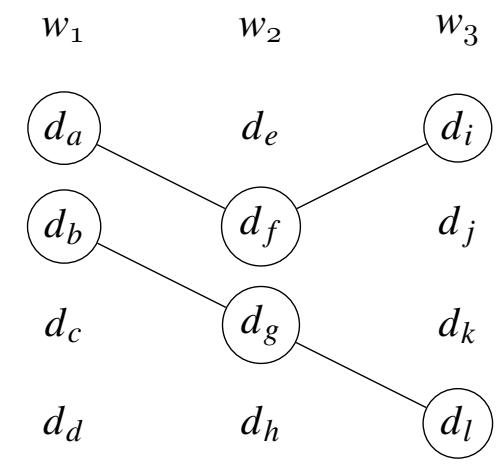

Figure 3: More than one Susan

The analysis of names as sorts thus retains the successes of the standard theory of names, while making room for situations in which more than one person bears the same name, such as the situations in which (21) and (22) would be uttered. This approach thus appears to be quite promising independent of -ever FRs.

Going back to -ever FRs, our analysis of names as sorts predicts the facts we observed in section 2.1. Recall that an -ever FR is infelicitous when the speaker is in a position to ascribe a proper name to the entity satisfying the content of the FR (Case A). This is predicted by Condition G: if the speaker can ascribe a name to the entity satisfying the content of the FR, then the name is a sort $G$ that applies to the denotation of the FR in each of the relevant worlds. Condition $\mathrm{G}$ gives a unified account, then, of the fact that proper names and nominal predicates both count as identifying.

\subsection{Demonstrative Pronouns and Sorts}

The remaining puzzle is why a speaker who is in a position to refer to an entity with a demonstrative pronoun can nonetheless felicitously use an -ever FR (section 2.2, Case B). We saw earlier that this fact cannot be explained on the view that rigidity is identification, and non-identification is achieved under nonrigidity. We now show how this fact can be explained given our approach to identification, whereby identification is achieved only when a sort is established for the entity in question.

In his classic work on demonstratives, Kaplan (1989) observes that although demonstrative pronouns are sensitive to speaker demonstrations (or referential in- 
tentions) and encode locative features, none of this information is part of the intension of a demonstrative. Consider (24) in a context where there is a white statue near the speaker and a black statue farther away, and the speaker is pointing at the black statue.

(24) If someone switched the places of the two statues...

a. ... the thing that I would be pointing at would be white.

b. ...the thing that would be in that location would be white.

c. ... that would be white.

Intuitively, (24a) and (24b) are true, contrasting with the false (24c). If the intension of the demonstrative pronoun contained information about the speaker demonstration or the relevant locative feature, (24c) would have a true reading like (24a) or (24b).

On Kaplan's account, while contextual factors such as demonstrations and locations play a role in determining the denotation of the demonstrative pronoun, the pronouns contribute only an entity to the compositional semantics, and this entity does not interact with the compositional semantics. ${ }^{8}$ The intension of a demonstrative pronoun is a rigid individual concept that, in each relevant possible world, picks out the entity that is identical to the referent in the actual world.

In a Geach-Gupta model, however, it is not meaningful to talk about rigidity in the same way, since transworld identity is relativized to a nominal sort. Are we forced to the conclusion that a demonstrative pronoun, by virtue of its rigidity, specifies a sort for its referent? The answer is no. While it is indeed the case that the demonstrated entity corresponds to entities in other worlds that are of the same sort, the demonstrative pronoun does not specify what sort that is.

This allows us to have a denotation for demonstrative pronouns which is minimally different from Kaplan's, as illustrated in (25), one which does not specify the sort of the referent. Since a demonstrative pronoun guarantees that the entity denoted is the same entity across worlds, and - because of the nature of transworld identity in the Geach-Gupta model — this is only possible when there is a sort that applies to the corresponding entities in each world, this individual concept must belong to some sort or other. The way we represent the fact that the sort is not specified is by stating that the individual concept belongs to the grand union of all sorts in the language.

$\llbracket$ that $\rrbracket^{M, w *, g}$ (type $\left.\langle s, e\rangle\right): i$, that is

a. a free variable over individual concepts whose value is determined by a contextually provided assignment function.

b. $i(w *)$ is demonstrated by the speaker in the context of utterance.

c. $i$ is a member of the grand union of nominal sorts $S$ relativized to $w *$.

\footnotetext{
${ }^{8}$ More recent work on demonstratives has challenged Kaplan's view that demonstratives never interact with other elements in the compositional semantics. See King (2001) and Wolter (2006) for alternative approaches to demonstratives.
} 
To understand the proposal in (25) on an intuitive level, it is helpful to compare demonstrative pronouns with the personal pronouns he and she, which provide a sort, i.e. man or woman, as they presuppose that their referents are people. ${ }^{9}$ Remember that person (unlike thing) denotes a sort. Demonstrative pronouns, by contrast, lack any such presumption or entailment - in fact, the sort of the referent of a demonstrative pronoun is under-determined by both the linguistic material itself and by any associated gestures. For example, the interpreter of (26) must rely on contextual inference to decide whether that denotes a statue, a certain kind of marble, an art installation, or even the fact that a statue has been installed in a particular location.

(26) (Speaker gestures towards a corner of the room, containing a marble statue) That is interesting.

Furthermore, demonstrative pronouns differ from personal pronouns in that they occur as the subject of identificational sentences like (27), which are used in presentational contexts where the identity of an entity is precisely what is at issue (Heller and Wolter 2008). This is just what we would expect of a pronoun that does not in itself establish the sort of its referent.

a. That's a statue.

b. This is Kris.

The personal pronouns he and she cannot be used in the same kind of construction in presentational contexts. In (28) below, the first sentence sets up a presentational context, and a personal pronoun is subsequently infelicitous.

(28) I'd like to make introductions...

a....\#She is Kris.

b. ... This is Kris.

An account of demonstrative pronouns as not establishing sorts thus fits our intuitions about how demonstrative pronouns are used, while personal pronouns seem to refer to entities that have already been established as belonging to the sort man or woman.

Returning to -ever FRs, we have a new prediction: a speaker who is in a position to use he or she should not be able to use an -ever FR. This prediction is borne out. Suppose that Dana sees a woman come into the room with a pot of soup. Dana has never met the woman before and doesn't know anything about her except that she was the last person to arrive. If Dana can point out the woman, he cannot utter (29) (repeated from 2):

(29) Whoever arrived last at the party brought the soup.

\footnotetext{
${ }^{9}$ More precisely, the sorts provided are male person and female person. We abstract away from age-related distinctions such as boy vs. man, as well as from uses of personal pronouns to refer to honorary persons such as pets and vehicles.
} 
Condition G straightforwardly explains the infelicity of (29) in this context: because Dana can use a personal pronoun to refer to the entity denoted by the -ever FR, there is a sort G, namely woman, that applies to the denotation of the FR in each of Dana's epistemic alternatives.

The analysis of demonstrative pronouns within the Geach-Gupta framework completes our account of indeterminacy in -ever FRs. We have argued that uttering a demonstrative pronoun does not specify a sort for the referent, although the nature of the model requires that such a sort exists. The referents of personal pronouns, by contrast, do belong to a specified sort, i.e. man or woman. This gives us a straightforward explanation of the interaction of these pronouns with -ever FRs. Remember that the indeterminacy requirement for for ignorance -ever FRs is with respect to the speaker's epistemic alternatives. A speaker who can use a demonstrative pronoun has not specified a sort for the denotation of the pronoun. Although she knows that in principle there is a sort that applies to the referent of the demonstrative in the world of utterance and to each of the corresponding entities in other worlds, this sort is not determined in her epistemic alternatives. Therefore, there is no $\mathrm{G}$ that applies to the denotation of the -ever FR in each of the relevant worlds, and the speaker is correctly predicted to be able to use an -ever FR for an entity that the speaker can refer to with a demonstrative pronoun. A speaker who can use he or she, on the other hand, has an established sort for the referent of the pronoun, which precludes her use of an -ever FR to refer to this entity.

\section{Conclusion}

In this paper we have provided a new definition of the indeterminacy requirement on -ever FRs, which requires non-identification of the entity denoted in some set of possible worlds. In particular, we have proposed that an -ever FR can be used felicitously by a speaker just in case there is no sort (in the sense of Gupta 1980) that is known by that speaker to apply to the entity denoted. In other words, we argue that identification in natural language is achieved when a sort is established for the entity in question, and thus an -ever FR requires indeterminacy with respect to sorts. We formalized this in Condition G. While we have focused here on ignorance readings of -ever FRs, we believe that this condition would naturally extend to other types of -ever FRs, as it represents a new generalization about what counts as identification in natural language.

The development of this condition has led us to conclude that a model where transworld identity is characterized by a single trivial principle of identity does not capture the nature of identity and identification in natural language. Instead, we have adopted a Geach-Gupta conception of transworld identity where the principles of transworld identity are many and non-trivial, and they are expressed in the meaning of nouns. This move has implications that go well beyond the meaning of -ever FRs, suggesting that it is time to reconsider the basic assumptions underlying analyses of constructions that are sensitive to the nature of identification. 


\section{References}

Brown, Roger: 1965, 'How Shall Things Be Called?', in R. C. Oldfield and J. C. Marshall (eds.), Language, 82-92. Penguin, Harmondsworth.

Caponigro, Ivano: 2003, Free Not to Ask: On the Semantics of Free Relatives and Wh-Words Cross-linguistically, Doctoral Dissertation, University of California, Los Angeles.

Condoravdi, Cleo: 2008, 'Free Choice and Uncertainty'. Handout of talk given at the Workshop on Inferential Mechanisms and their Linguistic Manifestations, University of Kyoto.

Cruse, D. Alan: 1977, 'The Pragmatics of Lexical Specificity', Journal of Linguistics 13, 153-164.

Dayal, Veneeta: 1997, 'Free Relatives and Any: Identity and Free Choice Readings', in A. Lawson (ed.), Proceedings of SALT VII.

von Fintel, Kai: 2000, 'Whatever', in Proceedings of SALT X, 27-40.

Geach, P. T.: 1962, Reference and Generality. Cornell University Press, Ithaca, NY.

Geach, P. T.: 1972, Logic Matters. University of California Press, Berkeley.

Gunlogson, Christine: 2001, True to Form: Rising and Falling Declaratives as Questions in English, Doctoral Dissertation, University of California, Santa Cruz.

Gupta, Anil: 1980, The Logic of Common Nouns: An Investigation in Quantified Modal Logic. Yale University Press, New Haven, CT.

Heller, Daphna: 2005, Identity and Information: Semantic and Pragmatic Aspects of Specificational Sentences, Doctoral Dissertation, Rutgers.

Heller, Daphna and Lynsey Wolter: 2008, 'That is Rosa: Identificational Sentences as Intensional Predication', in A. Grønn (ed.), Proceedings of Sinn und Bedeutung 12. University of Oslo.

Jacobson, Pauline: 1995, 'On the Quantificational Force of English Free Relatives', in E. Bach, E. Jelinek, A. Kratzer, and B. H. Partee (eds.), Quantification in Natural Languages, 451-486. Kluwer, Dordrecht.

Kaplan, David: 1989, 'Demonstratives', in J. Almong, J. Perry, and H. Wettstein (eds.), Themes from Kaplan, 481-563. Oxford University Press, Oxford.

King, Jeffrey C.: 2001, Complex Demonstratives. MIT Press, Cambridge, MA.

Kripke, Saul A.: 1982, Naming and Necessity. Harvard University Press, Cambridge, MA.

Tredinnick, Victoria Ann: 2005, On the Semantics of Free Relatives with -ever, Doctoral Dissertation, University of Pennsylvania.

Wolter, Lynsey: 2006, That's That: The Semantics and Pragmatics of Demonstrative Noun Phrases, Doctoral Dissertation, University of California, Santa Cruz. 